\title{
Evaluation of FLT-PET-CT as an imaging biomarker of proliferation in primary breast cancer
}

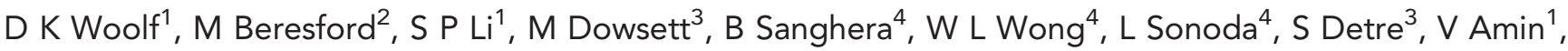 \\ M-L Ah-See ${ }^{1}$, D Miles ${ }^{1}$ and A Makris ${ }^{*}, 1$ \\ ${ }^{1}$ Breast Research Unit, Mount Vernon Cancer Centre, Rickmansworth Road, Northwood, HA6 2RN, UK; ${ }^{2}$ Department of Oncology, \\ Royal United Hospital Bath, Avon, Bath, BA1 3NG, UK; ${ }^{3}$ Academic Department of Biochemistry, Royal Marsden Hospital, Fulham \\ Road, London, SW3 6JJ, UK and ${ }^{4}$ Paul Strickland Scanner Centre, Mount Vernon Hospital, Rickmansworth Road, Northwood, \\ HA6 2RN, UK
}

Background: $\left[{ }^{18} \mathrm{~F}\right] f$ luorothymidine (FLT) has been proposed as a positron emission tomography (PET)-imaging biomarker of proliferation for breast cancer. The aim of this prospective study was to assess the feasibility of FLT-PET-CT as a technique for predicting the response to neoadjuvant chemotherapy (NAC) in primary breast cancer and to compare baseline FLT with Ki-67.

Methods: Twenty women with primary breast cancer had a baseline FLT-PET-CT scan that was repeated before the second cycle of chemotherapy. Expression of Ki-67 in the diagnostic biopsy was quantified. From the FLT-PET-CT scans lesion maximum and mean standardised uptake values $\left(\mathrm{SUV}_{\max }, \mathrm{SUV} V_{\text {mean }}\right)$ were calculated.

Results: Mean baseline SUV $\max$ was 7.3, and 4.62 post one cycle of NAC, representing a drop of 2.68 (36.3\%). There was no significant association between baseline, post chemotherapy, or change in SUV max and pathological response to NAC. There was a significant correlation between pre-chemotherapy Ki-67 and SUV $\max$ of $0.604(P=0.006)$.

Conclusions: Baseline SUV $\max$ measurements of FLT-PET-CT were significantly related to Ki-67 suggesting that it is a proliferation biomarker. However, in this series neither the baseline value nor the change in SUV $\max$ after one cycle of NAC were able to predict response as most patients had a sizeable SUV $\max$ reduction.

Over recent decades the routine addition of chemotherapy to the treatment paradigm for early breast cancer has improved mortality. Most chemotherapy is given in the adjuvant setting with the intention of treating occult micro-metastases and thereby improving survival. The use of chemotherapy in the neoadjuvant setting has the additional advantage of reducing the size of the primary tumour in order to facilitate breast-conserving surgery. Furthermore, it allows the study of the effects of chemotherapy on a primary breast carcinoma, using a combination of newer imaging techniques, as well as more conventional immunohistochemical and pathological measures, and to allow more accurate prognostication with the potential to individualise future patient therapies depending on initial response to treatment. $\left[{ }^{18} \mathrm{~F}\right]$ fluorothymidine (FLT) has been proposed as a suitable radiotracer for an imaging biomarker of proliferation with positron emission tomography (PET) (Shields et al, 1998). It is a fluorinemodified thymidine analogue that is related to cellular proliferation owing to its phosphorylation during the cell cycle S-phase by thymidine kinase-1, a pathway detailed by Buck et al (2009). A recently conducted systematic review and meta-analysis (Chalkidou et al, 2012) addressed the issue of the correlation between Ki-67 expression and FLT-PET, and concluded that there was significant correlation overall and in particular in brain, lung and breast cancer. Three studies, with a maximum of 15 patients, have looked specifically at the correlation between baseline FLT-PET and Ki-67 in patients with breast cancer with 
variable results (Smyczek-Gargya et al, 2004; Kenny, 2005; Contractor et al, 2011).

Pio et al (2006) investigated the predictive value of changes in FLT and FDG standardised uptake value (SUV) parameters before the initiation and after the first cycle of chemotherapy in patients with predominantly newly diagnosed metastatic breast cancer. Although changes in FLT SUV between the first two scans proved predictive of response, the study was not conducted in the neoadjuvant setting and did not address correlations with Ki-67, but rather the tumour marker CA27.29. Lubberink et al (2012) assessed the validity of simplified FLT uptake measures (standardised uptake values, tumour-to-blood ratios (TBRs)) in monitoring chemotherapy response (before and after the first cycle of chemotherapy) in patients with locally advanced breast cancer by comparing these against indices derived from pharmacokinetic modelling, which is the gold standard method for PET data analysis. Nevertheless, as the focus of the paper was primarily methodological, no correlations between pre- and post-therapy changes in FLT parameters with $\mathrm{Ki}-67$ and/or pathological response were derived, and hence no inferences on the predictive potential of changes in pre-/post-therapy FLT SUV measures were made. Therefore no study to our knowledge has addressed the vital issue of the change in FLT-PET parameters in response to chemotherapy and their baseline correlation to Ki-67.

Ki-67 has been consistently shown to be a prognostic marker in early breast cancer with 17 out of 18 studies with larger patient numbers showing statistical significance between Ki-67 levels and prognosis (Urruticoechea, 2005). A recent large study with 3658 evaluable patients has found $\mathrm{Ki}-67$ to be an independent predictor of both disease-free and overall survival (Inwald et al, 2013). It is also felt to have particular value as a measure of proliferation within clinical trials (Dowsett et al, 2011) and is considered by some to be a gold standard against which imaging results can be compared. Imaging biomarkers have advantages over traditional biomarkers such as Ki-67, in particular their non-invasive nature and ability to provide spatial data through both a primary tumour and local regional or metastatic disease. However, they require validation against a gold standard such as Ki-67.

The aim of this prospective study was to assess the feasibility of FLT-PET as a technique for predicting response to neoadjuvant chemotherapy (NAC) in operable breast cancer and to relate early changes in FLT uptake to final clinical and pathological response as well as obtain correlations with the proliferation marker Ki-67.

\section{MATERIALS AND METHODS}

Patients. Twenty women with newly diagnosed primary breast cancer were recruited into the study. All patients had biopsyproven breast cancer and had signed informed consent. The primary breast cancers were of clinical and/or radiological size $>2 \mathrm{~cm}$, and patients were WHO performance status 0 , or 1 , had adequate renal function, liver function and bone marrow function.

Study design. All patients were treated with a combination of 5-fluorouracil (500 $\left.\mathrm{mg} \mathrm{m}^{-2}\right)$, epirubicin $\left(75 \mathrm{mg} \mathrm{m}^{-2}\right)$ and cyclophosphamide $\left(500 \mathrm{mg} \mathrm{m}^{-2}\right)$ (FEC) or 5 -fluorouracil $\left(500 \mathrm{mg} \mathrm{m}^{-2}\right)$, epirubicin $\left(100 \mathrm{mg} \mathrm{m}^{-2}\right)$ and cyclophosphamide $\left(500 \mathrm{mg} \mathrm{m}^{-2}\right)$ followed by docetaxel $\left(100 \mathrm{mg} \mathrm{m}^{-2}\right.$ ) (FEC-T) chemotherapy by intravenous infusion on day 1 of a 21-day cycle. Neoadjuvant trastuzumab was not used, as it was not standard at the time of the study. Patients were planned to receive 6-8 cycles of FEC or FEC-T.

Patients had FLT-PET scans before commencing chemotherapy and at a median of 12 days (range 7-14) after the first dose (that is, before receiving the second cycle of treatment). In addition, 10 patients had a second ultrasound-guided biopsy performed 2 days after the second FLT-PET scan for evaluation of Ki- 67.
All patients gave written consent to participate in this study, which had approval from East and North Hertfordshire Local Research and Ethics Committee (04/Q0203/70) and the Administration of Radioactive Substances Advisory Committee (ARSAC), UK.

PET scanning protocol. FLT was synthesised by Hammersmith Imanet (GE Healthcare, Hammersmith Hospital, London, UK) and by Nottingham PETNET (Nottingham City Hospital, Nottingham, UK) by radiofluorination of the $2,3^{\prime}$-anhydro- $5^{\prime}$-O- $\left(4,4^{\prime}\right.$-dimethoxytrityl)-thymidine precursor using previously described methods (Cleij et al, 2012). Patients were weighed before each scan and were given an intravenous injection of $3.5 \mathrm{MBq} \mathrm{kg}^{-1}$ of FLT (maximum dose $350 \mathrm{MBq}$ ).

Clinical FLT-PET-CT scanning was performed with a GE Medical Systems (Hatfield, UK) Discovery ST machine. Scanning was initiated $90 \mathrm{~min}$ post injection allowing unbound (unphosphorylated) FLT to clear from normal tissue and equilibrium from blood to tissue and vice versa to take place, increasing the specificity of our measurements (Muzi et al, 2005). The CT scan started with a low-dose scout view to determine the axial range of measurement of the patient followed by a $140 \mathrm{kVp}, 80 \mathrm{~mA}$ helical scan. This scan was acquired before the PET acquisition for attenuation correction and to provide anatomic localisation. FLT uptake was calculated and recorded as SUV. Two-dimensional PET scanning (i.e., with septa in place) commenced immediately following the CT scan. For each subject the baseline scan involved a whole-body PET/CT scan covering the base of the brain (external auditory meatus) to pelvis, typically five bed positions acquired at $5 \mathrm{~min}$ per bed that is, typically a total of $25 \mathrm{~min}$. The second PET/ CT scan required only two bed positions to encompass the breasts lasting a total of $10 \mathrm{~min}$, allowing a reduction in radiation dose in comparison with whole-body scanning. PET data were reconstructed into a $128 \times 128$ matrix with a voxel size of $4.68 \times$ $4.68 \times 3.27 \mathrm{~mm}$, using the OSEM reconstruction algorithm (Hudson and Larkin, 1994) with two iterations and 30 subsets plus a 5-mm post-reconstruction Gaussian smoothing filter also applied. Corrections were applied for attenuation, scatter, random coincidences, isotope decay and dead time. Fused PET and CT images were subsequently generated. The average transverse and axial spatial resolution at $1 \mathrm{~cm}$ off-axis was $\sim 6.28$ and $6.88 \mathrm{~mm}$, respectively. No dietary restrictions were applied to the patients before imaging; patients were all scanned in the supine position and patients were informed of the importance of remaining static and having consistent shallow breathing throughout scanning.

Analysis of images. Two observers experienced in PET investigations and blinded to patient outcomes recorded the results individually. Standardised uptake values were derived from the radioactivity concentration in the tissue, the dose of radioactivity administered and the patient's weight. Both lesion maximum and mean SUV values ( $\mathrm{SUV}_{\max }$ and $\mathrm{SUV}_{\text {mean }}$, respectively) were obtained, along with tumour volume using vendor software that enabled positioning a three-dimensional volume of interest (VOI) to encompass the entire lesion. Standardised uptake value maximum within the tumour volume was determined from the VOI initially. Then the edge of the lesion for its entire volume was defined using isocontour thresholds of $30 \%, 40 \%, 50 \%$ and $60 \%$ $\mathrm{SUV}_{\max }$, respectively, and the mean value of the pixels within each derived volume was taken as $\mathrm{SUV}_{\text {mean }}$. This threshold method of determining the entire lesion mean SUV minimises observer bias in outlining regions of interest (ROI). In cases where the tumour was close to other high-uptake regions such as the liver or ribs, these areas could be edited out by hand to ensure they did not influence SUV readings. However, partial volume effects from nearby structures may still be evident. Standardised uptake value maximum and mean values were compared for pre-treatment and post-treatment scans and changes expressed as a percentage 
increase or decrease. These figures were then compared with clinical and pathological response measurements.

Tissue sampling and immunohistochemistry. The initial core biopsy performed at the time of diagnosis was analysed for Ki-67 as were the repeat biopsies after the first cycle of NAC where available. Four-micron-thick sections were cut onto glass slides (Thermo Scientific Superfrost Plus, Braunschweig, Germany). After drying at $37^{\circ} \mathrm{C}$ overnight, the sections were dewaxed in xylene and then rehydrated by immersing in decreasing concentrations of industrial-methylated spirits (IMS). The sections were then washed in tap water. Antigen retrieval was performed by microwaving the sections at $800 \mathrm{~W}$ for $10 \mathrm{~min}$ in preheated Dako low-pH Target Retrieval solution $\mathrm{pH}$ 6. Staining was conducted in a Dako Autostainer (Dako, Glostrup, Denmark) using the Dako REAL Detection System. Staining was performed using the MIB 1 primary antibody (Dako) at a dilution of 1 in 50. At the end of the autostainer run, the sections were removed from the instrument and counterstained with Mayer's Haematoxylin, dehydrated in increasing concentrations of IMS, cleared in xylene and mounted in DPX. A known positive Ki-67 control tissue was stained at the same time as the test sections, and in addition was run without primary antibody as the negative control. However the poor quality of the repeat biopsies performed after the first cycle of NAC did not allow further evaluation of this material.

Ki-67 visual scoring. The Ki-67 counting process was blinded to the results of the PET scan to avoid bias. Stained sections were examined visually using a Bright Field Microscope (Nikon UK, Kingston Upon Thames, UK) at $\times 400$ magnification using a $10 \times 10$-eyepiece graticule. The percentage of positive nuclei as a proportion of total number of invasive tumour cells was recorded in at least five random high-power fields (HPFs) representative of the proportions of stained and unstained tumour cells across the whole of the tumour. If 500 cells were not reached within the five HPFs, then more fields were counted until 500 cells achieved. The median Ki-67 score for the 10 fields was then calculated.

Measuring response. Clinical response was assessed before each cycle of chemotherapy. In addition, routine imaging was conducted during NAC at the clinicians discretion. Pathological response was assessed at definitive surgery on completion of NAC. A pathological complete response ( $\mathrm{pCR}$ ) was noted when there was no evidence of residual tumour on histological examination of the surgical specimen in the breast or lymph nodes. Patients with residual ductal carcinoma in-situ and no evidence of residual invasive disease were included in this category. Near pCR was defined as $>90 \%$ reduction in tumour cells, similar to the definition of Smith et al (2002). Patients were dicotomised into pathological responders (those who exhibited pCR or near pCR) and non-responders (all others).

Statistical analysis. Mean baseline and post-chemotherapy SUV $_{\text {max }}$ were correlated with pathological response using Pearson's correlation coefficient after tests for normality of the data were performed. Standardised uptake value mean data for isocontours $30,40,50$ and $60 \%$ were correlated to each other. The $40 \%$ isocontour (vendor software default) was used for further statistical analysis for consistency throughout this manuscript and has been shown to be of utility in other settings (Burri et al, 2008), although there is no agreed standard. Median Ki-67 values were compared with the mean and maximum SUV readings from the baseline FLT-PET and post-NAC scan in each patient. Simple linear regression was performed to obtain correlation coefficients for the two methods of measuring proliferation. Further exploratory analysis to evaluate the use of the total proliferative volume (TPV), total lesion proliferation (TLP) and TBRs were also performed. All statistical analyses were performed using SPSS statistical software (IBM Corp. Released 2012. IBM SPSS Statistics for Windows, Version 21.0. Armonk, NY, USA) and additional figures were produced with StatsDirect (StatsDirect Ltd., Altrincham, UK).

\section{RESULTS}

Twenty patients were successfully recruited to the study and completed the baseline FLT-PET-CT with 17 completing the second follow-up scan. Two patients declined to have the second FLT-PET-CT and one did not have NAC as detailed below. All 17 patients had a repeat FLT-PET-CT after a first cycle of FEC chemotherapy. Patients were recruited to the study from September 2005 to February 2006 and from December 2011 to December 2012; the pause in recruitment was due to a supply issue with the FLT tracer. Patient demographics and tumour characteristics are displayed in Table 1. The median age of the cohort was 52 (range 32-67), the median clinical tumour size was $6 \mathrm{~cm}$ (range $3-12 \mathrm{~cm}$ ) with the vast majority of tumours being invasive ductal carcinomas (95\%).

Twelve patients (60\%) received a combination of FEC and docetaxel, with one patient (5\%) additionally receiving cisplatin and gemcitabine owing to progressive disease in response to firstline treatment; other patients received FEC alone $(n=6,30 \%)$. One patient $(5 \%)$ received epirubicin and cyclophosphamide + accelerated paclitaxel. One patient (5\%) did not receive NAC owing to complications relating to cardiac hypoperfusion. The median number of cycles delivered was six (range 4-8).

Ten patients underwent mastectomy and 10 patients had a wide local excision. Fifteen patients had an axillary clearance with the remainder having upfront negative sentinel node biopsy. Of the 19

Table 1. Patient demographics and tumour characteristics

\begin{tabular}{|l|c|}
\hline Age (years) & $\mathbf{n = 2 0}$ \\
\hline Median & 51.8 \\
\hline Range & $31.6-67.0$ \\
\hline Inflammatory carcinoma & $2(10 \%)$ \\
\hline IDC & $19(95 \%)$ \\
\hline ILC & $1(5 \%)$ \\
\hline Grade 1 & $0(0 \%)$ \\
\hline Grade 2 & $10(50 \%)$ \\
\hline Grade 3 & $10(50 \%)$ \\
\hline T 1 & $0(0 \%)$ \\
\hline T 2 & $6(30 \%)$ \\
\hline T 3 & $12(60 \%)$ \\
\hline T 4 & $2(10 \%)$ \\
\hline N 0 & $8(40 \%)$ \\
\hline N 1 & $12(60 \%)$ \\
\hline N 2/3 & $0(0 \%)$ \\
\hline Triple negative & $6(30 \%)$ \\
\hline ER positive & $11(55 \%)$ \\
\hline PR positive & $10(50 \%)$ \\
\hline HER2 + ve & $6(30 \%)$ \\
\hline FEC & $6(30 \%)$ \\
\hline FEC-T & $13(65 \%)$ \\
\hline No chemotherapy & $1(5 \%)$ \\
\hline Abbreviations: ER=estrogen receptor; FEC=5-fluorouracil, epirubicin and cyclophospha- \\
mide chemotherapy; FEC-T=5-fluorouracil, epirubicin and cyclophosphamide followed by \\
docetaxel chemotherapy; IDC=invasive ductal carcinoma; ILC= invasive lobular carcinoma; \\
PR=progesterone receptor. & \\
\hline & \\
\hline
\end{tabular}


patients who underwent NAC, two (10.5\%) showed a pCR while five patients $(26 \%)$ demonstrated a near pCR. Six patients $(32 \%)$ demonstrated evidence of an effect from chemotherapy (partial pathological response-PR) and six patients (32\%) had no response to chemotherapy (NR). Seven out of the 15 patients (47\%) having an axillary dissection had pathologically involved lymph nodes with the remaining eight (53\%) having a pathologically negative axilla. Pathological response in the axilla was in the same category in all patients as pathological response in the primary tumour.

The median follow-up time was 22 months (range 5-90 months). Four patients (20\%) experienced a relapse, all with metastatic disease. There were four deaths, three (15\%) of which were related to metastatic breast cancer; however, one patient died due to complications relating to her breast cancer surgery.

Mean baseline $\mathrm{SUV}_{\max }$ was 7.3 (range 2.92-13.87). After one cycle of NAC mean $\mathrm{SUV}_{\max }$ was 4.86 (range 1.98-14.15) with a mean change of $-32.29 \%$ (range $11.29-62.51 \%$, 95\% CI -42.6 to $-20 \%, P=0.001$ ) (Figure 1). Fourteen patients (82\%) had a reduction in $\mathrm{SUV}_{\max }$ with $\mathrm{NAC}$, with the remaining three patients having an increase (range 2-11\%). Mean baseline SUV mean $40 \%$ was also calculated with a value of 4.105 (range 1.6-8.3). After one cycle of NAC, SUV mean was 2.72 (range 1-8.4) and a mean change in $\mathrm{SUV}_{\text {mean }}$ was $-33.40 \%$ (range $-60 \%$ to $8.82 \%$, 95\% CI -43.9 to $-22.1 \%, P=0.002$ ) (Table 2). Representative FLT-PET images are shown in Figure 2.

The mean change in $\mathrm{SUV}_{\max }$ of those having a pCR was $-31.47 \%$ (range -28.01 to -34.92 ), those having a near pCR was $-38.49 \%$ (range -0.49 to -62.51 ), PR was $-7.36 \%$ (range 11.29 to -50.95 ) and NR was $-44 \%$ (range -32.19 to -57.62 ). There was no significant association between pathological responders to NAC and $\mathrm{SUV}_{\max }$ at baseline $(r=0.103, P=0.674)$, post-NAC $(r=-0.098$, $P=0.71)$ or with change in $\mathrm{SUV}_{\max }(r=-0.151, P=0.563)$. The association between all responder categories and $\mathrm{SUV}_{\max }$ are shown in Supplementary Table S1.

Ki-67 data were available for 19 patients at baseline; there was insufficient histological material to score one baseline sample. Mean baseline Ki-67 was 32.2\% (range 2.3-68.4\%). Pearson's correlation showed a significant correlation between pre-chemotherapy Ki-67 and $\mathrm{SUV}_{\max }$ of $0.604(P=0.006)$ (See Figure 3) and $\mathrm{SUV}_{\text {mean }} 40 \%(r=0.64, P=0.003)$. There was also a nonsignificant association between $\mathrm{pCR}$ and higher baseline Ki-67 $(r=0.426, P=0.078)$.

$\mathrm{SUV}_{\text {mean }}$ data for 30, 40, 50 and $60 \%$ isocontours are presented in Figure 4 for both baseline and post one cycle of NAC. Pearson's correlation between all measures of SUV were high, ranging between 0.949 and $0.99(P<0.001)$ and showing good concordance between the measures (Table 3). The SUV mean data did not show a correlation with pathological response except for the association between partial response to chemotherapy and the change in $\mathrm{SUV}_{\text {mean }} 40 \%(r=0.59, P=0.013)$ (Supplementary Table S1).

Other PET parameters that were calculated and then tested for association against pathological response were TPV, TLP and TBR. The TPV is defined (Hatt et al, 2010) as the product of the tumour volume and the $\mathrm{SUV}_{\text {mean }}$, which we used at the $50 \%$ isocontour. The only significant association between TPV and pathological response was the change in TPV with a near pCR $(r=0.525$ $P=0.021$ ), although when the responders were grouped together this significant association was lost $(r=0.306, P=0.203)$ (Supplementary Table S2). We also calculated the TLP as the product of the functional tumour volume $\left(\mathrm{SUV}_{\text {mean }} 50 \%\right.$ tumour volume) and $50 \%$ of $\mathrm{SUV}_{\max }$ (Kahraman et al, 2012). This also showed a significant association between near $\mathrm{pCR}$ and change in TLP $(r=0.496, P=0.031)$ but again not when grouped into all responders $(r=0.283, P=0.241)$ (Supplementary Table S3). In order to calculate the TBR, we normalised tumour $\mathrm{SUV}_{\max }$ to arterial whole-blood SUV. Mean whole-blood SUVs were obtained by placing circular ROI in the middle of the aorta, calculating the $\mathrm{SUV}_{\text {mean }}$ in each individual slice and then averaging over 10 slices (Lubberink et al, 2012). No associations between TBR and pathological response were seen (Supplementary Table S4).

\section{DISCUSSION}

We have demonstrated that the majority of patients have a sizeable reduction in $\mathrm{SUV}_{\max }$ from a single cycle of NAC with a mean change of $-32.3 \%$. This study, however, failed to show any predictive markers of response after one cycle of chemotherapy. In terms of the histological proliferation biomarker Ki-67, we have shown a good correlation with FLT-PET pre-chemotherapy. The best predictive marker of response in terms of PCR was baseline Ki-67. These data therefore suggest that the main utility of FLT-PET as an imaging biomarker in early breast cancer is prechemotherapy, as a marker of proliferation, rather than in predicting pathological response after chemotherapy.

PET imaging is widely used in oncology and in breast cancer to image both primary and metastatic disease. It has many advantages including its reproducibility and non-invasive nature. It is also able to detect tumour heterogeneity, avoiding some of the sampling issues that are associated with histological analyses. Disadvantages include radiation dose, expense of the test, the need for expertise to perform and interpret the images, and the limited availability of some tracers including FLT (Murphy and Bergström, 2009), which is an issue we encountered on performing this study.
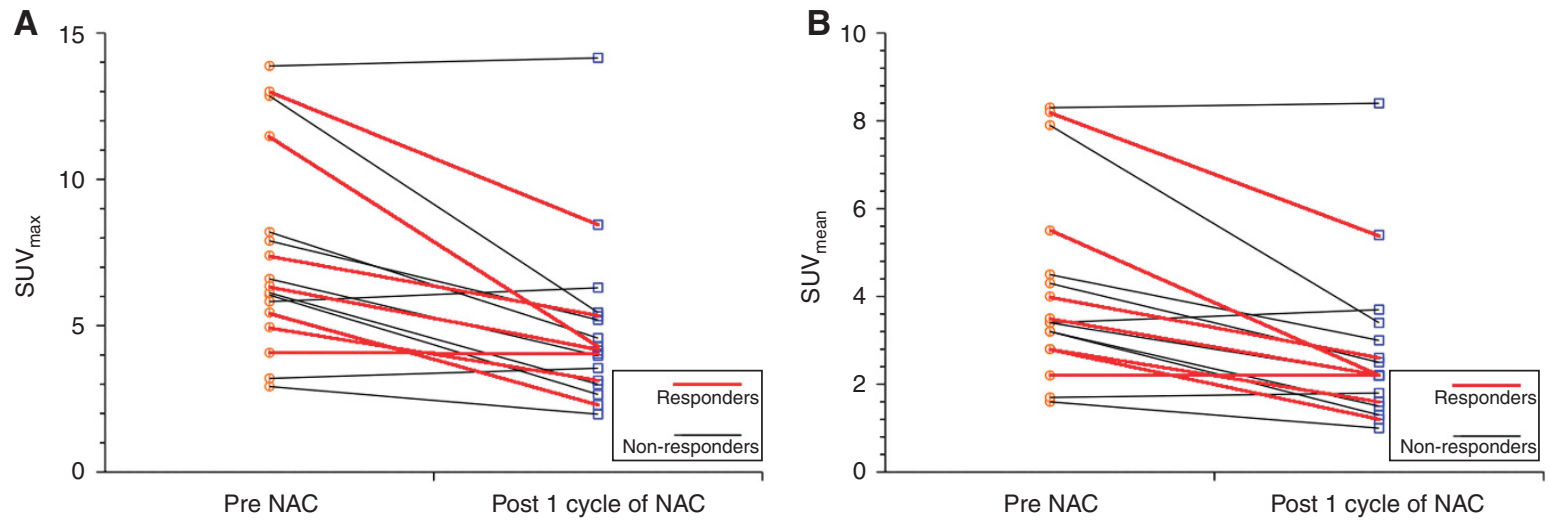

Figure 1. Ladder plots of (A) change in $\mathrm{SUV}_{\max }$ with NAC (B) change in $\mathrm{SUV}_{\text {mean }} 40 \%$ isocontour with NAC. Those achieving either a pCR or near PCR are classified as responders and all others as non-responders. 
Table 2. Ki-67, SUV $\max$ and SUV $V_{\text {mean }} 40 \%$ isodose values

\begin{tabular}{|c|c|c|c|c|c|c|c|c|}
\hline \multirow{2}{*}{ Patient no. } & \multirow[b]{2}{*}{ Path response } & \multirow[b]{2}{*}{$\begin{array}{c}\text { Ki-67 } \\
\text { Baseline }\end{array}$} & \multicolumn{3}{|c|}{ SUV $_{\max }$} & \multicolumn{3}{|c|}{$\mathrm{SUV}_{\text {mean }} 40 \%$ isodose } \\
\hline & & & Baseline & Post 1 cycle NAC & $\%$ change & Baseline & Post 1 cycle NAC & $\%$ change \\
\hline 1 & NR & 44.7 & 6.05 & 2.66 & -56.03 & 3.2 & 1.3 & -59.38 \\
\hline 2 & PR & 60.7 & 13.87 & 14.15 & 2.02 & 8.3 & 8.4 & 1.20 \\
\hline 3 & Near pCR & 21.1 & 11.47 & 4.3 & -62.51 & 5.5 & 2.2 & -60.00 \\
\hline 4 & NR & 2.3 & 2.92 & 1.98 & -32.19 & 1.6 & 1 & -37.50 \\
\hline 5 & NR & 9.2 & 6.59 & 3.98 & -39.61 & 3.4 & 2.2 & -35.29 \\
\hline 6 & Near pCR & 16.5 & 4.07 & 4.05 & -0.49 & 2.2 & 2.2 & 0 \\
\hline 7 & PR & $a$ & 3.19 & 3.55 & 11.29 & 1.7 & 1.8 & 5.88 \\
\hline 8 & $\mathrm{pCR}$ & 40.8 & 7.39 & 5.32 & -28.01 & 4 & 2.6 & -35.00 \\
\hline 9 & Near pCR & 23.8 & 4.96 & 3.13 & -36.90 & 2.8 & 1.6 & -42.86 \\
\hline 10 & NR & 28.8 & 7.91 & 5.2 & -34.26 & 4.5 & 3 & -33.33 \\
\hline 11 & PR & 13.7 & 5.83 & 6.3 & 8.06 & 3.4 & 3.7 & 8.82 \\
\hline 12 & PR & 32.4 & 6.116 & 3 & -50.95 & 3.2 & 1.5 & -53.13 \\
\hline 13 & $P R$ & 16 & 3.53 & a & a & 1.9 & $\mathrm{a}$ & a \\
\hline 14 & NR & 29.8 & 12.86 & 5.45 & -57.62 & 7.9 & 3.4 & -56.96 \\
\hline 15 & Near $p C R$ & 42.6 & 6.36 & 4.16 & -34.59 & 3.5 & 2.2 & -37.14 \\
\hline 16 & PR & 22.1 & 4.8 & a & $\mathrm{a}$ & 2.5 & $a$ & $a$ \\
\hline 17 & Near pCR & 54.1 & 5.45 & 2.3 & -57.80 & 2.8 & 1.2 & -57.14 \\
\hline 18 & a & 68.4 & 11.44 & $a$ & a & 7.2 & a & $\mathrm{a}$ \\
\hline 19 & $\mathrm{pCR}$ & 59.5 & 13 & 8.46 & -34.92 & 8.2 & 5.4 & -34.15 \\
\hline 20 & NR & 25.8 & 8.21 & 4.57 & -44.34 & 4.3 & 2.5 & -41.86 \\
\hline
\end{tabular}

A

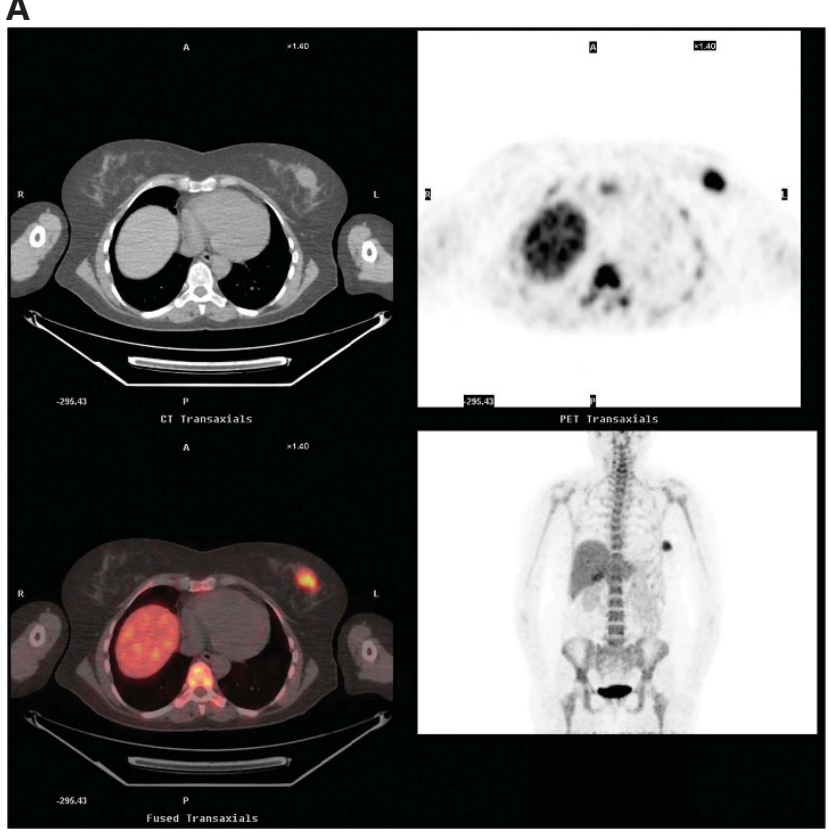

B

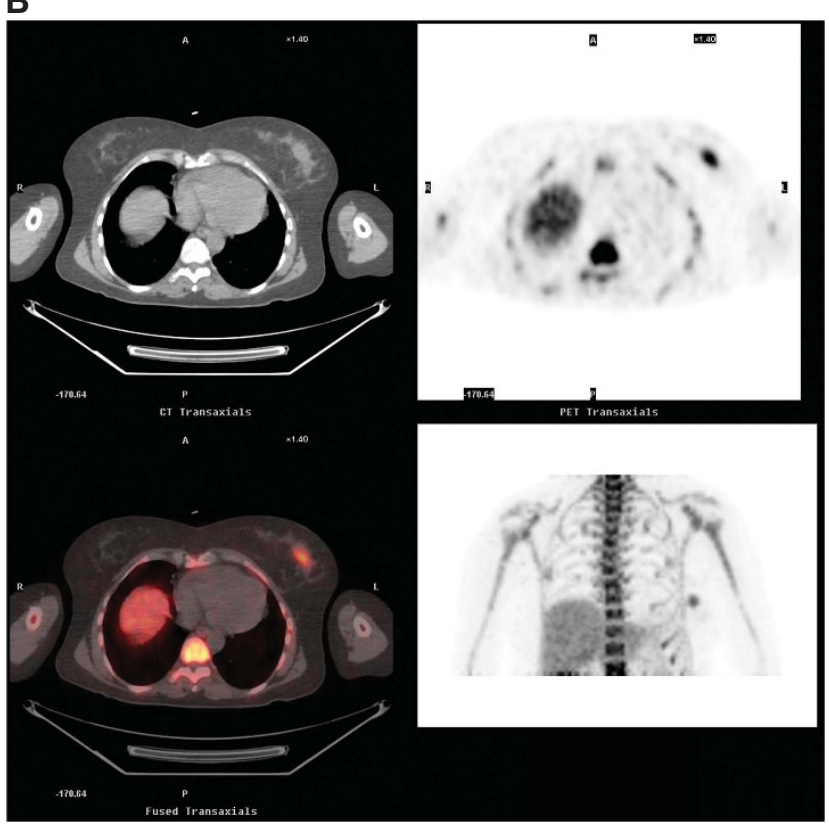

Figure 2. FLT-PET images representing SUV $\max (300$ dpi CT, PET, colour-fused and MIP images) for a 55-year-old female presenting with a 7-cm ER + ve, PR - ve, Her 2 + ve breast cancer (A) pre-chemotherapy SUV max $_{\text {of }} 13$ and Ki-67 of 59.5 (B) post 1 cycle of FEC with a SUV max $_{\text {max }} 8.5$ ( $-35 \%)$. She went on to have a pathological CR and is alive and disease free at a follow-up of 16 months. 
In terms of prediction of response to therapy our study failed to demonstrate any utility to FLT-PET. Only four patients (24\%) failed to have an imaging response as defined by a reduction in $\mathrm{SUV}_{\text {max }}$ or $\mathrm{SUV}_{\text {mean }}$ ( $40 \%$ isocontour), of which three had a slight increase in SUV, with 11 patients (65\%) having a reduction of

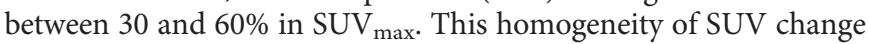
in response to NAC demonstrates the difficulty in the differentiation of responding and non-responding patients. This is evidenced by our data showing that the mean change in $\mathrm{SUV}_{\max }$ for those with a pCR was $-31.47 \%$, which was less than the mean change of those experiencing a near pCR at $-38.49 \%$. Previous studies have shown utility to the FLT-PET parameters TPV (Hatt et al, 2010), TLP (Larson et al, 1999; Gulec et al, 2011; Kahraman et al, 2011) and TBR (Lubberink et al, 2012). Our further analysis of these parameters did show significance for the first two of these against near $\mathrm{pCR}$, but there was no durable pattern to this finding and it could be owing to multiplicity of statistical analysis.

Our data are consistent with the literature where, to our knowledge, only two other reports have successfully demonstrated any form of clinical benefit associated with change in FLT values with treatment. Zander et al (2011) and Kahraman et al (2011), in a phase II clinical trial in 34 patients with advanced non-small cell lung cancer treated with the tyrosine kinase inhibitor erlotinib, showed that an early (1 week post treatment) FLT response predicted for prolonged progression-free survival. However, both

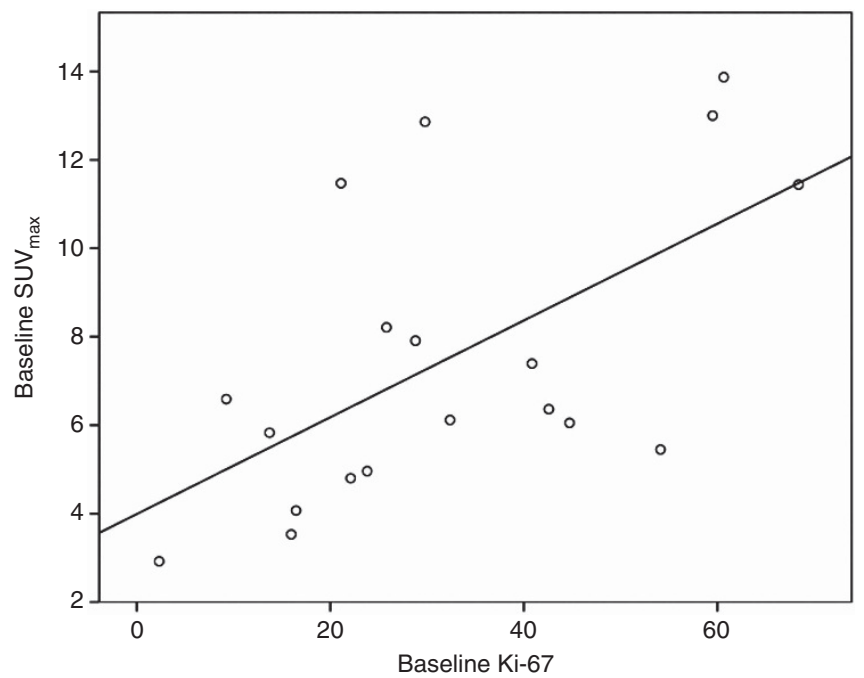

Figure 3. Correlation of baseline $\mathrm{Ki}-67$ and $\mathrm{SUV}_{\max }$ with line of best fit $(r=0.604, P=0.006)$.

A

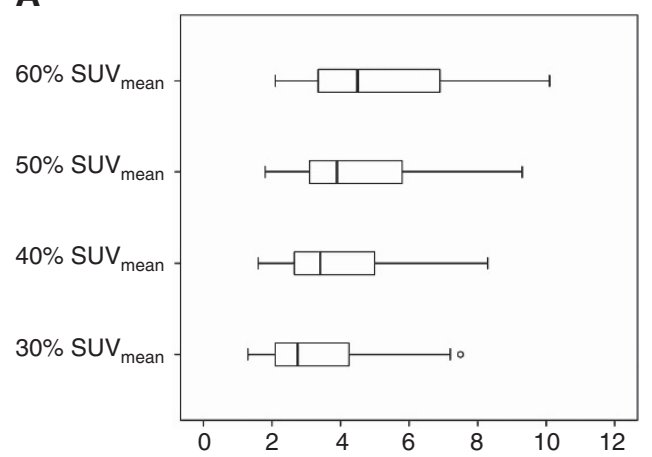

reports failed to show any difference in overall survival, or any predictive factor after 6 weeks of treatment. The same study also evaluated FDG PET, which performed better with successful prediction of non-progression, progression-free survival and overall survival in the same patients.

Prior breast cancer data have also shown a correlation between FLT-PET parameters and Ki-67 in studies of 12 and 8 patients (Kenny, 2005; Contractor et al, 2011), but there is also conflicting data in a study of 12 patients that failed to show a correlation (Smyczek-Gargya et al, 2004). Some of these studies included data on lymph nodes as well as distant metastasis. A meta-analysis of 27 studies in a variety of tumour types has shown a strong correlation between Ki-67 and FLT uptake $(r=0.7, P<0.001)$ (Chalkidou et al, 2012) in keeping with the findings of our study. A large review of the predictive role of $\mathrm{Ki}-67$ in NAC has concluded that that $\mathrm{Ki}-67$ is a predictive marker for both clinical and pathological response (Yerushalmi et al, 2010). A high level of Ki-67 pre-NAC was considered to be a predictor for response to chemotherapy as we have also shown in our data, but is also associated with poorer long-term outcomes. This is a well-described paradox (Weigel and Dowsett, 2010), where those who have a high Ki-67 have a better chance of achieving a pCR and therefore better long-term outcomes, but if they do not achieve a pCR they have a particularly poor outcome (Jones et al, 2008). Our data have suggested a correlation between a high Ki-67 level at baseline and pCR, although this failed to meet statistical significance $(P=0.078)$, perhaps partly owing to the small numbers in the pCR group in this study (10.5\%). FLT-PET data from other non-breast cancer studies have also demonstrated a correlation between FLT uptake and Ki-67 index (Tehrani and Shields, 2013), displaying that this is not a phenomena exclusive to breast carcinomas.

\begin{tabular}{|c|c|c|c|c|c|}
\hline $\begin{array}{l}\text { Correlation } \\
\text { of change in } \\
\text { SUVs }\end{array}$ & SUV $_{\text {max }}$ & $\begin{array}{c}\text { SUV }_{\text {mean }} \\
30 \%\end{array}$ & $\begin{array}{c}S_{\text {SUV }} \\
40 \%\end{array}$ & $\begin{array}{c}\text { SUV }_{\text {mean }} \\
50 \%\end{array}$ & $\begin{array}{c}S^{S U V} V_{\text {mean }} \\
60 \%\end{array}$ \\
\hline$S U V_{\max }$ & NA & $0.982^{a}$ & $0.99^{a}$ & $0.992^{a}$ & $0.979^{a}$ \\
\hline$S U V_{\text {mean }} 30 \%$ & $0.982^{a}$ & NA & $0.99^{a}$ & $0.976^{a}$ & $0.949^{a}$ \\
\hline$S U V_{\text {mean }} 40 \%$ & $0.99^{a}$ & $0.99^{\mathrm{a}}$ & NA & $0.993^{a}$ & $0.971^{a}$ \\
\hline$S U V_{\text {mean }} 50 \%$ & $0.992^{\mathrm{a}}$ & $0.976^{a}$ & $0.993^{a}$ & NA & $0.983^{a}$ \\
\hline$S U V_{\text {mean }} 60 \%$ & $0.979^{a}$ & $0.949^{a}$ & $0.971^{\mathrm{a}}$ & $0.983^{a}$ & NA \\
\hline
\end{tabular}

B

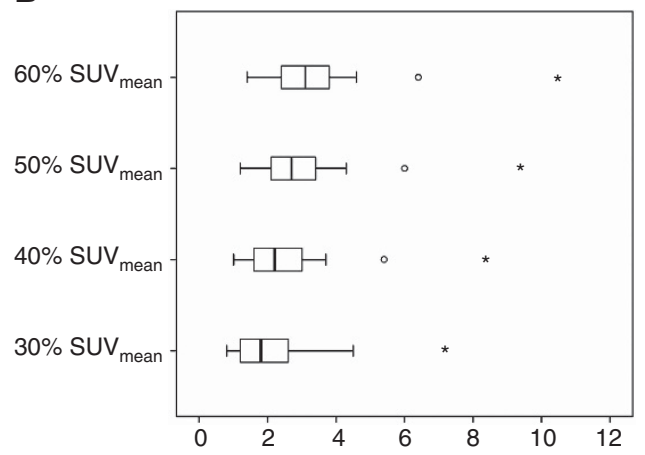

Figure 4. Box and whisker plot of SUV mean for 30,40,50, and 60\% isocontours. Whiskers represent range, box represents interquartile range, line represents median, ${ }^{\circ}$ indicate outliers and * indicate extreme outliers. (A) Pre-NAC and (B) post 1 cycle of NAC. 
In addition to assessing the lesion $\mathrm{SUV}_{\max }$, which is the most widely reported measure for FLT-PET, we also reported the results of lesion $\mathrm{SUV}_{\text {mean }}$. We found an excellent correlation between these measures, but other data suggest that the choice of SUV measure used strongly affects the ability to correctly classify patients into treatment responder categories (i.e., responder and non-responder) (Vanderhoek et al, 2013). In this study, SUV mean were derived from the entire lesion volume for different isocontour thresholds, thereby reducing potential bias of higher $\mathrm{SUV}_{\text {mean }}$ values arising when only a single slice in the lesion incorporating $\mathrm{SUV}_{\max }$ is used for analysis. In order for FLT-PET to become more widely used, it is important to successfully establish the optimal threshold and/or additional parameters for its measurement. In fact, when compared with FDG PET, FLT is likely to have a lower SUV reading as well as a lower range (Buck et al, 2003; Yap et al, 2006; Kasper et al, 2007). This reduced quantitative parameter will have an impact on the ability to establish useful cutoff data for responders and non-responders.

The mechanism underpinning the decrease in FLT uptake in response to therapy is thought to be thymidylate synthase inhibition, which is a vital enzyme involved in the de novo synthesis of DNA (Perumal, 2006). The use of different therapeutic agents may affect FLT uptake regardless of the direct effect on proliferation depending on whether the agent affects nucleoside transport or metabolism (Tehrani and Shields, 2013) and the phase of the cell cycle in which it operates (Vallabhajosula, 2007), and this has been shown in human breast cancer cells (Direcks et al, 2008). 5-FU has been shown to decrease FLT uptake, doxorubicin increases FLT uptake and paclitaxel has minimal effect. All of the patients in our study were exposed to the same chemotherapy agents before the second FLT-PET-CT scan being performed, so this issue is unlikely to be a confounding factor within our patients, but may have implications if one was to generalise our results to other combinations of chemotherapy agents. The same study (Direcks et al, 2008) also showed a change in FLT uptake over time, and as our patients were not all imaged at exactly the same interval post chemotherapy this may have an implication on our results.

Our study did not involve any use of reproducibility imaging, although this has been addressed by previous FLT studies in the settings of non-small cell lung cancer, head and neck cancer, as well as breast cancer. These overall have shown good reproducibility with Shields et al (2008) showing the SUV mean to be highly correlated between the first and the second scan $\left(r^{2}=0.99\right.$, $P<0.0001)$, Cheebsumon et al (2011) showing the test-retest variability for metabolic volume to range between 7.4 and $29 \%$ (similar to that of FDG PET from the same study), Langen et al (2008) showing reproducibility of over 0.9 on most parameters and Kenny et al (2007) showing a test-retest correlation coefficient of $\geqslant 0.97$. All of these studies suggest that sequential FLT-PET-CT scans are reproducible in patients before the receipt of chemotherapy, adding weight to our findings of a consistent reduction in SUVs in response to treatment.

There are some cautions to the interpretation of our results. The numbers in this study were small, but consistent with other similar studies. Poor-quality repeat biopsies did not allow us to correlate changes in FLT SUV max $_{\text {max }}$ with change in Ki-67. There are also no fixed guidelines on how to define response in FLT-PET, although the PERCIST criteria may become established in the future (Wahl et al, 2009), so we were unable to define and classify imaging responders.

Our study has shown that baseline Ki-67 and FLT SUV max $_{\text {is }}$ well correlated in keeping with FLT-PETs status as a proliferation biomarker, although Ki-67 had a better predictive ability in terms of pathological outcome. The situation post NAC is more complex where our study failed to show any predictive ability of either absolute or change in FLT SUV $\max$ or other PET parameters.

\section{ACKNOWLEDGEMENTS}

We are grateful to both the Breast Cancer Campaign and the Breast Cancer Research Trust for funding this study.

\section{CONFLICT OF INTEREST}

The authors declare no conflict of interest.

\section{REFERENCES}

Buck AK, Halter G, Schirrmeister H, Kotzerke J, Wurziger I, Glatting G, Mattfeldt T, Neumaier B, Reske SN, Hetzel M (2003) Imaging proliferation in lung tumors with PET: 18F-FLT versus 18F-FDG. J Nucl Med 44: 1426-1431.

Buck AK, Herrmann K, Shen C, Dechow T, Schwaiger M, Wester H-J (2009) Molecular imaging of proliferation in vivo: positron emission tomography with [18F]fluorothymidine. Methods 48: 205-215.

Burri RJ, Rangaswamy B, Kostakoglu L, Hoch B, Genden EM, Som PM, Kao J (2008) Correlation of positron emission tomography standard uptake value and pathologic specimen size in cancer of the head and neck. Int J Radiat Oncol Biol Phys 71: 682-688.

Chalkidou A, Landau DB, Odell EW, Cornelius VR, O’Doherty MJ, Marsden PK (2012) Correlation between Ki-67 immunohistochemistry and 18F-Fluorothymidine uptake in patients with cancer: a systematic review and meta-analysis. Eur J Cancer 48: 3499-3513.

Cheebsumon P, van Velden FHP, Yaqub M, Frings V, de Langen AJ, Hoekstra OS, Lammertsma AA, Boellaard R (2011) Effects of image characteristics on performance of tumor delineation methods: a test-retest assessment. J Nucl Med 52: 1550-1558.

Cleij MC, Steel CJ, Brady F, Ell PJ, Pike VW, Luthra SK (2012) An improved synthesis of $3^{\prime}$-DEOXY-3'-[18F]fluorothymidine ([18F]FLT) and the fate of the precursor, 2, 3'-anhydro-5'-O-(4, 4'-dimethoxytrityl)-thymidine. J Label Compd Radiopharm 44: S871-S873.

Contractor KB, Kenny LM, Stebbing J, Challapalli A, Al-Nahhas A, Palmieri C, Shousha S, Lewis JS, Hogben K, De Nguyen Q, Coombes RC, Aboagye EO (2011) Biological basis of $\left[{ }^{11} \mathrm{C}\right]$ cholinepositron emission tomography in patients with breast cancer: comparison with $\left[{ }^{18} \mathrm{~F}\right]$ fluorothymidine positron emission tomography. Nucl Med Commun 32: 997-1004.

Direcks WGE, Berndsen SC, Proost N, Peters GJ, Balzarini J, Spreeuwenberg MD, Lammertsma AA, Molthoff CFM (2008) [18F]FDG and [18F]FLT uptake in human breast cancer cells in relation to the effects of chemotherapy: an in vitro study. Br J Cancer 99: 481-487.

Dowsett M, Nielsen TO, A'Hern R, Bartlett J, Coombes RC, Cuzick J, Ellis M, Henry NL, Hugh JC, Lively T, McShane L, Paik S, Penault-Llorca F, Prudkin L, Regan M, Salter J, Sotiriou C, Smith IE, Viale G, Zujewski JA, Hayes DF (2011) Assessment of Ki67 in breast cancer: recommendations from the International Ki67 in Breast Cancer Working Group. J Natl Cancer Inst 103: 1656-1664.

Gulec SA, Suthar RR, Barot TC, Pennington K (2011) The prognostic value of functional tumor volume and total lesion glycolysis in patients with colorectal cancer liver metastases undergoing $90 \mathrm{Y}$ selective internal radiation therapy plus chemotherapy. Eur J Nucl Med Mol Imaging 38: 1289-1295.

Hatt M, Cheze-Le Rest C, Aboagye EO, Kenny LM, Rosso L, Turkheimer FE, Albarghach NM, Metges JP, Pradier O, Visvikis D (2010) Reproducibility of $18 \mathrm{~F}-\mathrm{FDG}$ and $3^{\prime}$-Deoxy-3'-18F-fluorothymidine PET tumor volume measurements. J Nucl Med 51: 1368-1376.

Hudson HM, Larkin RS (1994) Accelerated image reconstruction using ordered subsets of projection data. IEEE Trans Med Imaging 13: 601-609.

Inwald EC, Klinkhammer-Schalke M, Hofstädter F, Zeman F, Koller M, Gerstenhauer M, Ortmann O (2013) Ki-67 is a prognostic parameter in breast cancer patients: results of a large population-based cohort of a cancer registry. Breast Cancer Res Treat 139: 539-552.

Jones RL, Salter J, A'Hern R, Nerurkar A, Parton M, Reis-Filho JS, Smith IE, Dowsett M (2008) The prognostic significance of Ki67 before and after neoadjuvant chemotherapy in breast cancer. Breast Cancer Res Treat 116 : 53-68. 
Kahraman D, Holstein A, Scheffler M, Zander T, Nogova L, Lammertsma AA, Boellaard R, Neumaier B, Dietlein M, Wolf J, Kobe C (2012) Tumor lesion glycolysis and tumor lesion proliferation for response prediction and prognostic differentiation in patients with advanced non-small cell lung cancer treated with erlotinib. Clin Nucl Med 37: 1058-1064.

Kahraman D, Scheffler M, Zander T, Nogova L, Lammertsma AA, Boellaard R, Neumaier B, Ullrich RT, Holstein A, Dietlein M, Wolf J, Kobe C (2011) Quantitative analysis of response to treatment with erlotinib in advanced non-small cell lung cancer using 18F-FDG and 3“-Deoxy-3-"18F-fluorothymidine PET. J Nucl Med 52: 1871-1877.

Kasper B, Egerer G, Gronkowski M, Haufe S, Lehnert T, Eisenhut M, Mechtersheimer G, Ho AD, Haberkorn U (2007) Functional diagnosis of residual lymphomas after radiochemotherapy with positron emission tomography comparing FDG- and FLT-PET. Leuk Lymphoma 48: 746-753.

Kenny L, Coombes RC, Vigushin DM, Al-Nahhas A, Shousha S, Aboagye EO (2007) Imaging early changes in proliferation at 1 week post chemotherapy: a pilot study in breast cancer patients with $3^{\prime}$-deoxy- $3^{\prime}$ [18F]fluorothymidine positron emission tomography. Eur J Nucl Med Mol Imaging 34: 1339-1347.

Kenny LM (2005) Quantification of cellular proliferation in tumor and normal tissues of patients with breast cancer by [18F]fluorothymidinepositron emission tomography imaging: evaluation of analytical methods. Cancer Res 65: 10104-10112.

Langen AJ, Klabbers B, Lubberink M, Boellaard R, Spreeuwenberg MD, Slotman BJ, Bree R, Smit EF, Hoekstra OS, Lammertsma AA (2008) Reproducibility of quantitative $18 \mathrm{~F}-3^{\prime}$-deoxy- $3^{\prime}$-fluorothymidine measurements using positron emission tomography. Eur J Nucl Med Mol Imaging 36: 389-395.

Larson SM, Erdi Y, Akhurst T, Mazumdar M, Macapinlac HA, Finn RD, Casilla C, Fazzari M, Srivastava N, Yeung HWD, Humm JL, Guillem J, Downey R, Karpeh M, Cohen AE, Ginsberg R (1999) Tumor treatment response based on visual and quantitative changes in global tumor glycolysis using PET-FDG imaging. the visual response score and the change in total lesion glycolysis. Clin Positron Imaging 2: 159-171.

Lubberink M, Direcks W, Emmering J, Tinteren H, Hoekstra OS, Hoeven JJ, Molthoff CFM, Lammertsma AA (2012) Validity of simplified 3'-deoxy$3^{\prime}-[18 \mathrm{~F}]$ fluorothymidine uptake measures for monitoring response to chemotherapy in locally advanced breast cancer. Mol Imaging Biol 14: 777-782.

Murphy PS, Bergström M (2009) Radiopharmaceuticals for oncology drug development: a pharmaceutical industry perspective. Curr Pharm Des 15 957-965.

Muzi M, Vesselle H, Grierson JR, Mankoff DA, Schmidt RA, Peterson L, Wells JM, Krohn KA (2005) Kinetic analysis of $3^{\prime}$-deoxy- $3^{\prime}$-fluorothymidine PET studies: validation studies in patients with lung cancer. J Nucl Med 46: 274-282.

Perumal M (2006) Redistribution of nucleoside transporters to the cell membrane provides a novel approach for imaging thymidylate synthase inhibition by positron emission tomography. Cancer Res 66: 8558-8564.

Pio BS, Park CK, Pietras R, Hsueh W-A, Satyamurthy N, Pegram MD, Czernin J, Phelps ME, Silverman DHS (2006) Usefulness of 3'-[F18] fluoro- $3^{\prime}$-deoxythymidine with positron emission tomography in predicting breast cancer response to therapy. Mol Imaging Biol 8: 36-42.
Shields AF, Grierson JR, Dohmen BM, Machulla HJ, Stayanoff JC, Lawhorn-Crews JM, Obradovich JE, Muzik O, Mangner TJ (1998) Imaging proliferation in vivo with [F-18]FLT and positron emission tomography. Nat Med 4: 1334-1336.

Shields AF, Lawhorn-Crews JM, Briston DA, Zalzala S, Gadgeel S, Douglas KA, Mangner TJ, Heilbrun LK, Muzik O (2008) Analysis and reproducibility of $3^{\prime}$-deoxy- $3^{\prime}$-[18F]fluorothymidine positron emission tomography imaging in patients with non-small cell lung cancer. Clin Cancer Res 14: 4463-4468.

Smith IC, Heys SD, Hutcheon AW, Miller ID, Payne S, Gilbert FJ, Ah-See AK, Eremin O, Walker LG, Sarkar TK, Eggleton SP, Ogston KN (2002) Neoadjuvant chemotherapy in breast cancer: significantly enhanced response with docetaxel. J Clin Oncol 20: 1456-1466.

Smyczek-Gargya B, Fersis N, Dittmann H, Vogel U, Reischl G, Machulla H-J, Wallwiener D, Bares R, Dohmen BM (2004) PET with [18 F]fluorothymidine for imaging of primary breast cancer: a pilot study. Eur J Nucl Med Mol Imaging 31: 720-724.

Tehrani OS, Shields AF (2013) PET imaging of proliferation with pyrimidines. J Nucl Med 54: 903-912.

Urruticoechea A (2005) Proliferation marker Ki-67 in early breast cancer. J Clin Oncol 23: 7212-7220.

Vallabhajosula S (2007) 18F-labeled positron emission tomographic radiopharmaceuticals in oncology: an overview of radiochemistry and mechanisms of tumor localization. Semin Nucl Med 37: 400-419.

Vanderhoek M, Perlman SB, Jeraj R (2013) Impact of different standardized uptake value measures on PET-based quantification of treatment response. J Nucl Med 54: 1188-1194.

Wahl RL, Jacene H, Kasamon Y, Lodge MA (2009) From RECIST to PERCIST: evolving considerations for PET response criteria in solid tumors. J Nucl Med 50: 122S-150S.

Weigel MT, Dowsett M (2010) Current and emerging biomarkers in breast cancer: prognosis and prediction. Endocr Relat Cancer 17: R245-R262.

Yap CS, Czernin J, Fishbein MC, Cameron RB, Schiepers C, Phelps ME, Weber WA (2006) Evaluation of thoracic tumors with 18Ffluorothymidine and 18F-fluorodeoxyglucose-positron emission tomography. Chest 129: 393-401.

Yerushalmi R, Woods R, Ravdin PM, Hayes MM, Gelmon KA (2010) Ki67 in breast cancer: prognostic and predictive potential. Lancet Oncol 11: 174-183.

Zander T, Scheffler M, Nogova L, Kobe C, Engel-Riedel W, Hellmich M, Papachristou I, Toepelt K, Draube A, Heukamp L, Buettner R, Ko YD, Ullrich RT, Smit E, Boellaard R, Lammertsma AA, Hallek M, Jacobs AH, Schlesinger A, Schulte K, Querings S, Stoelben E, Neumaier B, Thomas RK, Dietlein M, Wolf J (2011) Early prediction of nonprogression in advanced non-small-cell lung cancer treated with erlotinib by using [18F]fluorodeoxyglucose and [18F]fluorothymidine positron emission tomography. J Clin Oncol 29: 1701-1708.

This work is published under the standard license to publish agreement. After 12 months the work will become freely available and the license terms will switch to a Creative Commons AttributionNonCommercial-Share Alike 3.0 Unported License.

Supplementary Information accompanies this paper on British Journal of Cancer website (http://www.nature.com/bjc) 